\title{
Contents, Vol. 49, 1923
}

Inhalts -Verzeichnis.

Eigen-Arbeiten. $\quad$ seite

Bab, W., Zur Frage der Zuverlässigkeit der Tonometer . 17 Birch-Hirschfeldt, A., Zur Bedeutung und Messung der

Lidbulbusspannung 79

Blasl·ovics, L. v., Über die Entstehung des senilen Ek-

und Entropiums 30

- $\quad$ - Eine Operation zur Beseitigung des Entropium

habituale 95

Blatt, N., Zur Kasuistik der Augenveränderungen bei

Vergiftung durch Schlangenbiß 280

Blessig, E., Zur Statistik der schweren Augenverletzungen

im Frieden und im Kriege 193

Gzellitzer, A., Augenarzt und Erbkunde 335

Echeverria, M., Über pseudosympathisierende Entzündung 100 Erlanger, G., Zur Therapie und Atiologie der Keratitis

disciformis. Schneller Heilungserfolg mit Ionto-

phorese 118

Fietta, Über eine seltene Form von Geschwürsbildung in

der Sklera 90

Fuchs, A. und E. Lauda, Zur Atiologie der Keratitis den-

dritica 9

Guist, G., Über ein Instrument zur Messung von Höhen-

verdrängungen der Augen 189

Handmann, M., Über voreilige Tätowierung der Netzhaut 5 Heßberg, R., Über die Bebandlung ekzematöser Augen-

erkrankungen nach Deycke-Much 327

Knüsel, 0. und P. Vonwiller, Vitale Färbungen am mensch-

lichen Auge. (Hierzu Tafel I-IV) 157.

Kramer, R., Zur Atiologie der Dacryocystitis congenita . 20 Kraupa, E.y Linsenkapselrisse ohne Wundstar .... 93 Metier, J., Über spontane Heilung von Netzhautgangliom 1

- $\quad$ - Über Pseudosarcoma chorioideae 235

Meyerhof, M. $\uparrow$ Die Bewertung der bei Grippe vorkommen-

den Augenmuskellähmungen 208

Nowak, E., Über das Ponndorf-Verfahren bei den phlyktä-

nulären Augenerkrankungen 24

Pillat, A., Über die gittrige und andere Formen degene-

rativer Hornhauterkrankungen 313

Salzer, F., Über Hornhautregeneration und ein neues Ver- 
fahren der Abrasio corneae, die Keratektomie . . . 283

$-\mathrm{IV}-$

Seite

Schinck, Ein Fall von Stauungspapille bei Thrombopenie.Ein Beitrag zur Frage der

Papillenveränderungen beiden hämorrhagischen Diathesen 265

Schwarzkopf, G., Über die Plasmazelle und das Plasmom

der Konjunktiven 247

Mitteilungen aus der Praxis für die Praxis.

Engelbrecht, W., Über einen Fall von Starkstromverletzung

mit beiderseitiger Katarakt 345

Friede, R., Zur Technik der Kataraktextraktion .... 347

Bericht über die deutscne ophthalmologische Literatur.

Untersuchungsmethoden (I. u. II. Semester 1921). Von

Dr. R. Kramer in Wien

124

Gesellschaftsberichte.

Versammlung deutscher Naturforscher und Ärzte in Leipzig. Abteilung: Augenheilkunde.

Bericht von Dr.

Dieter in Leipzig 42

Gemeinsame Sitzungen der Wiener biologischen und ophthalmologischen Gesellschaft vom 13., 20. und

27. März 1922134

Ophthalmologische Gesellschaft in Wien. Sitzungen vom

2. V., 22. V. und 19. V. $1922 \ldots \quad 216$

Sitzungen vom 10. XII. und 23. X. 1922 .... 291

Sitzung vom 20. XL $1922 \quad 349$

Gesellschaft für Wissenschaft und Leben im Rheinisch-Westfälischen Industriebezirk.

Ophthalmologische Abteilung. Bericht von Dr. Heßberg in Essen.

Sitzung vom 2. Mai $1922 \quad 52$

Sitzung vom 28. X. $1922 \quad 299$

XIV. Jahresversammlung der Ungarischen Ophthalmologischen Gesellschaft in Budapest am 3.-

5. Juni 1922.

Bericht von Prof. Dr. v. Liebermann 57

Sitzung der medizinischen Gesellschaft Basel am 6. Juli 192264

Diagnose und Therapie 69, 144, 228, 307, 364

Bericht über den Spaltlampenmikroskopieknrs der Uni-

versitäts-Augenklinik in Basel

Buchbespreehungen 154, 234

P. Personalien 156

312

Tagesnachrichteii 312 